\title{
Modification of Concrete Damaged Plasticity model. Part I: Modified plastic potential
}

\author{
Aleksander Szwed $^{1, *}$, and Inez Kamińska ${ }^{1}$ \\ ${ }^{1}$ Warsaw University of Technology, Al. Armii Ludowej 16, 00-637 Warszawa, Poland
}

\begin{abstract}
In order to extend the Concrete Damaged Plasticity model [1], a modification of flow rule is considered. A new plastic potential is proposed. It takes into account the difference in behavior of concrete under compressive and tensile loading. In the present paper the proposed function is examined and compared to the original potential. Influence of parameters of the potential choice on the shape of its surface is analysed. Formulation and preliminary validation of the modified model is shown in the subsequent paper (Part II).
\end{abstract}

\section{Introduction}

Experiments show that prediction of the response of concrete structures subjected to the loading exceeding plastic limit should take into account its elastic and plastic behaviour as well as degradation of elastic stiffness due to plastic deformation and initiation of cracks. Basic models for concrete and soil use Drucker-Prager type functions as both the yield condition and the plastic potential [1-7]. The experiments showing deviation of plastic strain rate tensor from the gradient of yield function are scarce, compare [8] for soils, however it is frequently assumed that the flow rule should be non-associative $[5,6,8]$. Isotropic damage described by one scalar parameter is often accepted as a rough approximation but also models taking into account anisotropic damage of material are developed [9].

The basic model for numerical computation implemented in Abaqus [1] was proposed by Lubliner [6] and further developed by Lee and Fenves [5]. It is formulated in the effective stress space, contains Lubliner's yield condition and the non-associative flow rule, as well as a scalar damage parameter. Material damage evolves along with the plastic strain growth; however the coupling between damage and plasticity is weak. The model has been continuously modified to better fit experimental data [7,10,11]. The main features taken into consideration by the researchers are the hardening rule and the failure criterion $[10,11]$. In the present study other refinement of this elastoplastic damage model for concrete is presented - an improved plastic potential is proposed.

The plastic potential introduced in this paper takes into account different behavior of concrete under the compressive and tensile loading. Two conic surfaces, according to the Lubliner's yield condition or the two-surface Drucker-Prager condition, devoted to the tension and compression zones respectively, are used as the envelope for definition of

* Corresponding author: a.szwed@il.pw.edu.pl 
a new plastic potential. The proposed potential is smooth, so its gradient used in the flow rule is uniquely defined. Basic four material parameters involved in the definition of function can be calibrated on the basis of results of four experiments: the uniaxial tension and compression, the equal biaxial compression and the triaxial compression tests. Additional four free parameters used in the definition of potential can be calibrated to fit observed behavior of material. Including plasticity condition and other equations, the model requires input of twelve parameters and four material functions. Regarded modification of the existing CDP Abaqus model can improve performance of the model to fit a wider range of loading regimes observed in concrete structures. Plastic flow defined by the proposed function can be calibrated independently for the tension and compression zone of loading. Attempts of calibration of the CDP model have been made in the literature e.g. [12-14].

In Part I, the difference between the original Abaqus potential based on two free parameters and the proposed potential is shown, as well as typical angle of deviation of direction of the plastic flow from the normal to the yield surface. The influence of change of model parameters on the shape of potential's surface is investigated. Subsequent paper (Part II) deals with the model formulation and computations for the case of uniaxial loading.

\section{Plastic potential}

The Drucker-Prager type potentials are commonly used to cover observed performance of concrete structures, compare [1-7] among others. They characterize properly compressive behavior of the material though they fail to model its tensile properties. As an extension, the two-surface Drucker-Prager condition should be used to properly portray the compressive and tensile behavior of material. However, in this case discontinuity of the gradient of plastic potential at the joint of two surfaces is observed. The proposed potential is intended to overcome this inconvenience and to extend flexibility of calibration of the model. The function is based on the selected meridional sections of Lubliner's yield condition [6] determined separately for the tension $\left(\sigma_{\max }=\sigma_{1}>0\right)$ and compression $\left(\sigma_{1} \leq 0\right)$. It is smooth thus the flow rule is properly defined for all points of the stress space.

The envelope for the plastic potential (meridians of the Lubliner's condition) is defined as follows:

$$
f(r, \xi)=\left\{\begin{array}{lll}
\xi+\alpha_{1} r-\beta_{1}=0 & \text { for } & \xi_{W} \geq \xi \geq \xi_{P}, \\
\xi+\alpha_{2} r-\beta_{2}=0 & \text { for } \quad \xi<\xi_{P},
\end{array}\right.
$$

where:

$$
\begin{gathered}
\xi=\frac{1}{\sqrt{3}} \operatorname{tr} \boldsymbol{\sigma}, \quad r=\sqrt{\operatorname{trs}^{2}} \\
\alpha_{1}=\frac{3+2 m_{1} \beta}{\sqrt{2}(3 \alpha+\beta)}, \quad \beta_{1}=\frac{\sqrt{3}(1-\alpha)}{3 \alpha+\beta} \sigma_{C}, \quad \alpha_{2}=\frac{3+2 m_{2} \gamma}{\sqrt{2}(3 \alpha+\gamma)}, \quad \beta_{2}=\frac{\sqrt{3}(1-\alpha)}{3 \alpha+\gamma} \sigma_{C}, \\
\alpha=\frac{\sigma_{B C}-\sigma_{C 0}}{2 \sigma_{B C}-\sigma_{C 0}}, \quad \beta=(1-\alpha) \frac{\sigma_{C}}{\sigma_{T}}-(1+\alpha), \quad \gamma=-(1-\alpha) \frac{\sigma_{C 0}}{\sigma_{T C}}-(2+\eta) \alpha+\eta-1, \\
\xi_{P}=\frac{2 \sqrt{3}(1-\alpha)\left(m_{1} \beta-m_{2} \gamma\right)}{3\left(1-2 m_{2} \alpha\right) \gamma-3\left(1-2 m_{1} \alpha\right) \beta+2\left(m_{1}-m_{2}\right) \gamma \beta} \sigma_{C}, \quad \xi_{W}=\frac{\sqrt{3}(1-\alpha)}{3 \alpha+\beta} \sigma_{C}=\beta_{1} .
\end{gathered}
$$


Notations $\sigma_{C 0}, \sigma_{T 0}$ and $\sigma_{B C}$ stand for the initial compressive yield stress, the tensile yield limit and the biaxial compressive yield stress respectively ( $\sigma_{C}$ and $\sigma_{T}$ are the current values), and $\eta \sigma_{T C}$ is the triaxial compressive yield limit in a test where the stress tensor has representation $\boldsymbol{\sigma} \rightarrow-\sigma_{C T} \operatorname{diag}[1,1, \eta]$, compare [15]. Both $m_{1}=\cos \theta_{1}$ and $m_{2}=\cos \theta_{2}$ are parameters describing selected meridians of the Lubliner's condition. Angles $\theta_{1}$ and $\theta_{2}$ belong to the interval $\langle 0, \pi / 3\rangle$, so the parameters $m_{1}, m_{2} \in\langle 0.5,1.0\rangle$. If both asymptotes are located on the tensile meridian then $m_{1}=m_{2}=1.0$, and on the compressive meridian $m_{1}=m_{2}=0.5$. The proposed range for $m_{1}$ and $m_{2}$ parameters can be widened when the calibration of the model requires it.

The first formula in (1) is applicable for the tension zone while the second applies to the compressive zone.

To avoid discontinuity of the gradient and to properly portray both the tensile and compressive behavior of concrete the following plastic potential is proposed:

$$
\Phi(\boldsymbol{\sigma})=\xi-f_{s}(r),
$$

where $f_{s}$ is a function of the stress deviator invariant $r$ :

$$
\begin{gathered}
f_{s}(r)=\frac{1}{2}\left(f_{1 n}+f_{2 n}-\sqrt{\left(m_{4} A\right)^{2}+\left(f_{1 n}-f_{2 n}\right)^{2}}\right), \\
f_{1 n}(r)=\beta_{1}-\sqrt{A^{2}+\left(\alpha_{1} r\right)^{2}}, f_{2 n}(r)=\beta_{2}-\sqrt{A^{2}+\left(\alpha_{2} r\right)^{2}} \\
A=\frac{2\left(1-m_{3}\right) \beta_{1}\left(\beta_{2}-m_{3} \beta_{1}\right)}{\beta_{1}+\beta_{2}-2 m_{3} \beta_{1}+\sqrt{\left(\beta_{2}-\beta_{1}\right)^{2}+m_{4}^{2} \beta_{1}\left(\beta_{2}-m_{3} \beta_{1}\right)\left(1-m_{3}\right)}} .
\end{gathered}
$$

$m_{3} \in(0,1)$ is a parameter characterizing location of the vertex of the plastic potential surface. It describes the closeness of the apex to the point where envelope $(1)_{1}$ intersects axis $\xi$, compare Fig. 1(a) and Fig. 2(c). Parameter $m_{4}>0$ is chosen arbitrarily to properly model the vertex region and the transition zone from meridian $\theta_{1}$ to $\theta_{2}$ associated with the branches of envelope (1). It influences strongly the curvature of surface (3) in the region near the potential apex - the derivative changes rapidly for great values of $m_{4}$, while for smaller values the change is gradual, see Fig. 1(b) and Fig. 2(d).

The asymptote for meridional section of (3) is the compressive cone (1) 2 . As it is depicted on Fig.1(b), the potential's derivative with respect to $r$ is positive for whole domain, which results in the curve showing its meridional section being a growing function of $r$. In the vertex point, $\xi=m_{3} \xi_{W}$, the derivative equals 0 , so then the potential is smooth for $r=0$.

It is worth mentioning that during plastic hardening (or softening) the values of $\beta, A$, $\alpha_{1}, \beta_{1}$ and $\beta_{2}$ change as the development of plastic limits occurs, compare equations (2). The values of $\alpha, \gamma$ and $\alpha_{2}$ remain constant. 
(a)

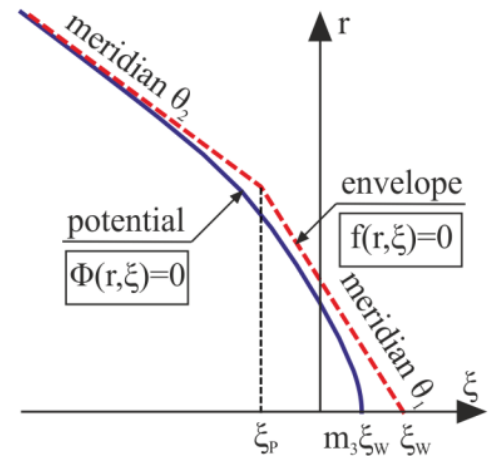

(b)

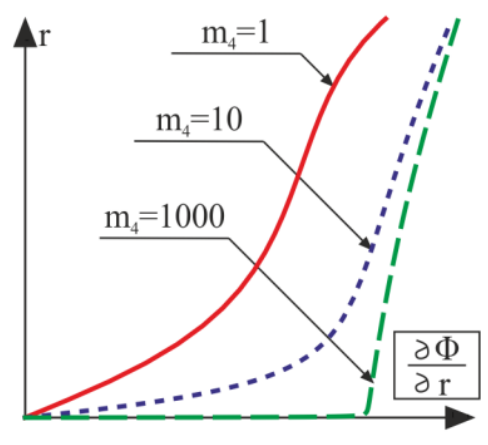

Fig. 1. The proposed potential: (a) meridional section and envelope, (b) derivative of the potential due to variable $r$ for various values of parameter $m_{4}$.
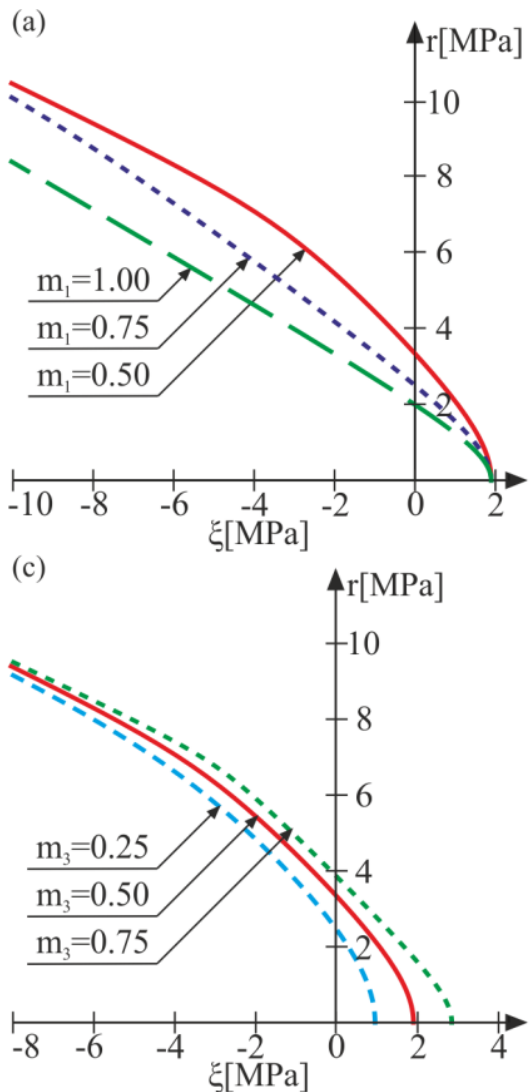
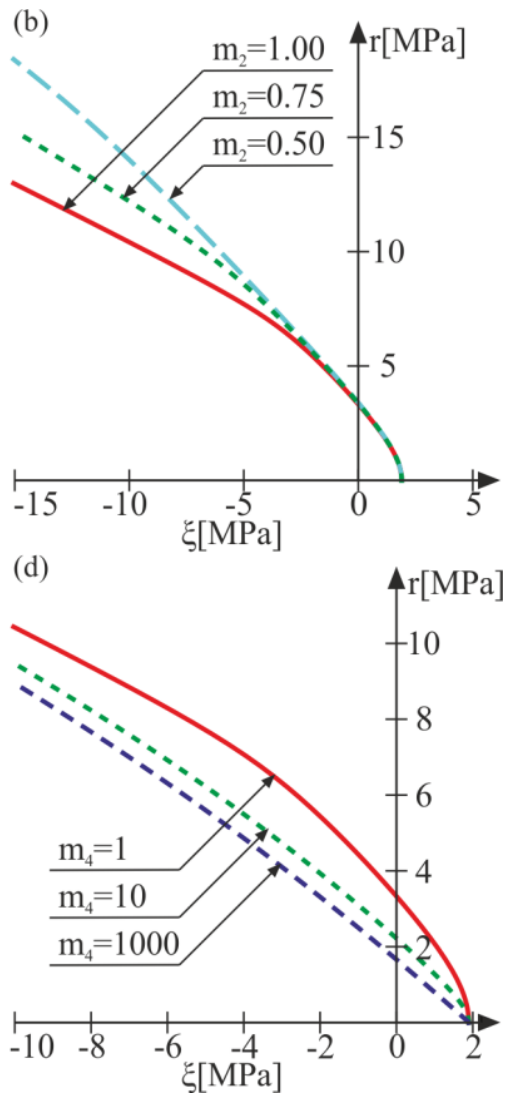

Fig. 2. Meridional section of the plastic potential for several values of: (a) $m_{1}$, (b) $m_{2}$, (c) $m_{3}$, (d) $m_{4} ; \sigma_{C 0}=20 M P a, \sigma_{T 0}=2 M P a, \sigma_{B C}=23 M P a, \eta \sigma_{T C}=123 M P a$.

In comparison to the original plastic potential [1], the proposed surface (3) offers possibility of distinguishing the compressive and tensile stress states, see Fig. 3(a). Gradient of the primary function has approximately the same direction for almost all $r$, while the modified plastic potential changes the slope when passing from the compressive to the tensile states, as it is shown in Fig. 3(b). Potential (3) allows to adjust the direction of 
plastic flow through change of the parameters $m_{1}$ and $m_{2}$ responsible for the slope of meridians, see Fig. 2(a),(b) and Fig. 3(b). For the plastic potential to be convex one needs to ensure $\alpha_{1} \leq \alpha_{2}$, which imposes a condition on values of $m_{1}$ and $m_{2}$, see equations (2).

The proposed potential (3) imitates the yield condition, however its normal differs from the normal of the yield surface. The deviation of gradient of the plastic potential from the normal to the yield surface is approximately constant along chosen meridians of the Lubliner's condition, see Fig. 4. Sudden changes appear in the zone of transition between the compressive and tensile states (i.e. passing through $\sigma_{\max }=\sigma_{1}=0$ plane), and close to the vertex zone.

(a)

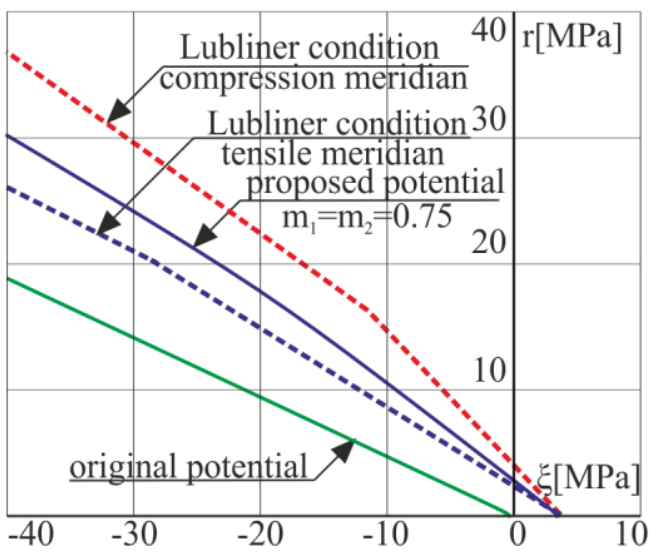

(b)

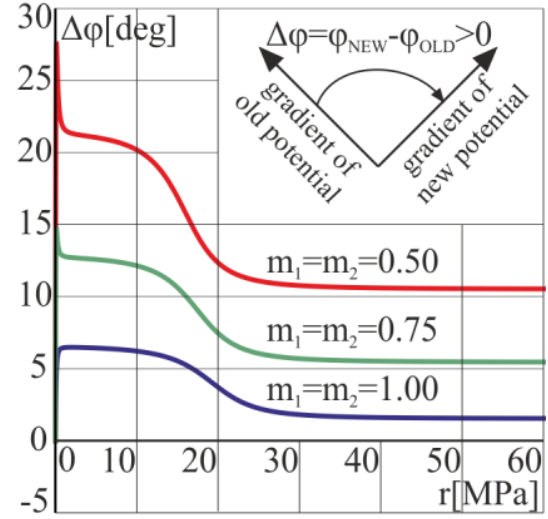

Fig. 3 The proposed potential versus original potential for: $\sigma_{C 0}=20 M P a, \sigma_{T 0}=2 M P a$, $\sigma_{B C}=23 \mathrm{MPa}, \eta \sigma_{T C}=123 \mathrm{MPa}, m_{3}=0.9$, and $\phi=45^{\circ}, \beta_{M}=0.1$ [1]; (a) meridional section, (b) the difference of directions (angles) of the potential's gradients in relation to $r$ axis.
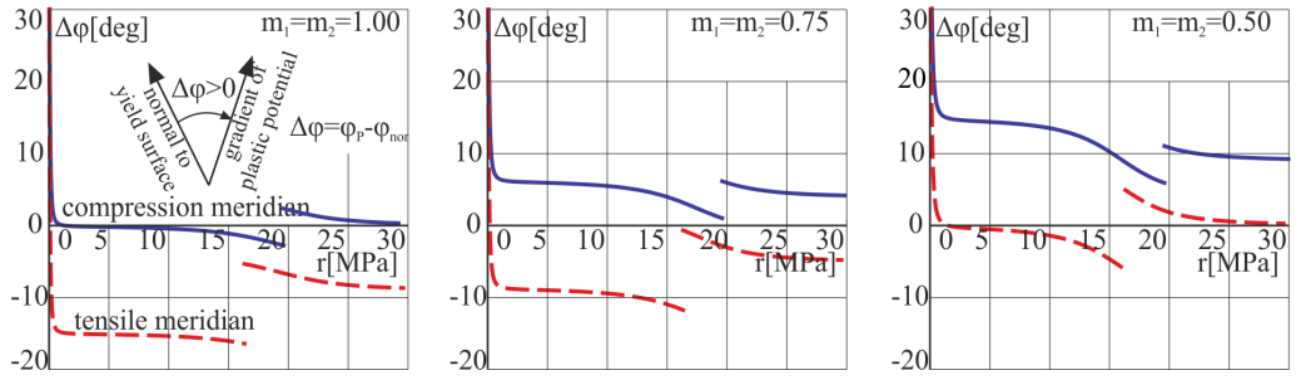

Fig. 4 Angle between normal to the plastic surface and gradient of the proposed potential for various parameters $m_{1}$ and $m_{2}$ - meridional section for: $\sigma_{C 0}=20 M P a, \sigma_{T 0}=2 M P a, \sigma_{B C}=23 M P a$, $\eta \sigma_{T C}=123 \mathrm{MPa}, m_{3}=0.9, m_{4}=10$.

\section{Conclusions}

A new plastic potential for the refined elastoplastic model for damaged material allows for distinguishing the tensile and the compressive stress states which is of great importance when describing concrete, rock and soil behavior. Free parameters $m_{1}$ and $m_{2}$ permit of adjusting the potential to the chosen slopes of meridians of the Lubliner's yield condition. The deviation of direction of the plastic flow gradient from the yield surface gradient can be 
easily handled by selecting adequate coefficients. Parameter $m_{3}$ governs location of the vertex of plastic potential, while parameter $m_{4}$ models smoothness of the potential in a zone surrounding the apex, as well as the smoothness of zone of transition from the compressive to the tensile states. In original Abaqus CDP model only two free parameters for the slope (dilation angle) and smoothness at the vertex (eccentricity) are available.

Parameters of plastic potential are to be calibrated on basis of four material tests: the uniaxial tension and compression, biaxial uniform compression and triaxial compression. Omitting this assumption the new potential can be reduced to the original one introducing:

$$
\alpha_{1}=\alpha_{2}=\frac{3}{\sqrt{2} \operatorname{tg} \psi}, A=\sqrt{3} \beta_{M} \sigma_{T 0}, \quad \beta_{1}=\beta_{2}=\frac{1}{2} m_{4} A,
$$

where $\beta_{M}$ is eccentricity and $\psi$ - dilation angle defined for the original potential [1].

A possible improvement of the function concerns the deviatoric section of potential surface - it is reasonable to introduce the dependency of plastic potential of the third stress invariant [15-17]. Dependence of plastic potential on the third invariant can enhance flexibility of the model and effectively control the non-associative flow in deviatoric crosssection. Extensive study on defining the shape of deviatoric cross-section in plasticity surfaces is presented in [17] and other literature.

\section{References}

\section{Abaqus Theory Manual}

2. R. Eid, P. Pautre, Eng. Struct. 29, 3301 (2007)

3. J. Jiang, Y. Wu, Int. J. Solids Struct. 49, 445 (2012)

4. W. B. Kratzig, R. Polling, Comput. Struct. 82, 1201 (2004)

5. J. Lee, G. Fenves, Earthquake Eng. Struct. Dyn. 27, 937 (1998)

6. J. Lubliner, J. Oliver, S. Oller, E. Onate, Int. J. Solids Struct. 25(3), 299 (1989)

7. O. Omidi, V. Lofti, Earthquake Eng. Struct. Dyn. 42, 2129 (2013)

8. J. Lee, G. Fenves, Earthquake Eng. Struct. Dyn. 27, 937 (1998)

9. S. Murakami, Continuum Damage Mechanics (Springer, 2012)

10. N. J. Hany, E. G. Hantouche, M. H. Harajli, Eng. Struct. 125, 1 (2016)

11. Z. Tao, Z. Wang, Q. Yu, J. Constr. Steel Res. 89, 121 (2013)

12. I. Kamińska, A. Szwed, Concrete and concrete structure - experiments, E. Szmigiera, P. Łukowski, S. Jemioło (eds.), 93 (OWPW, Warsaw, 2015) (in Polish)

13. P. Kmiecik, M. Kamiński, Archives of Civil and Mechanical Engineering XI(3), 623 (2011)

14. M. Szczecina, A. Winnicki, ASEM15 (Korea, 2015)

15. N. S. Ottosen, ASCE J. Eng. Mech. Div. 103, 527 (1977)

16. A. Szwed, Failure criteria and constitutive equations of material with degradation, $\mathrm{Ph}$. D. Dissertation (Warsaw, 2000) (in Polish)

17. A. Szwed, Deformation and Stregth of Materials and Structures, S. Jemioło, A. Szwed (eds.) 3, 51 (Warsaw, 2013) (in Polish) 